\title{
The Optical Variability of the BL Lac AO 0235+164
}

\author{
Junhui Fan 1,2,*, Omar Kurtanidze ${ }^{3,4}$, Yi Liu ${ }^{1,2}$, Xiang Liu ${ }^{5}$, Gotthard M. Richter ${ }^{6}$, \\ Maria G. Nikolashvili ${ }^{3}$, Sophia O. Kurtanidze ${ }^{3}$, Revaz Chanishvili ${ }^{3}$, Hongtao Wang ${ }^{7}$, \\ Mahito Sasada ${ }^{8}$, Aiying Zhou ${ }^{9}$, Chao Lin ${ }^{1,2}$ and Yuhai Yuan ${ }^{1,2}$ \\ 1 Center for Astrophysics, Guangzhou University, Guangzhou 510006, China; pinux@gzhu.edu.cn (Y.L.); \\ 374848511@qq.com (C.L.); yh_yuan@gzhu.edu.cn (Y.H.Y.) \\ 2 Astronomy Science and Technology Research Laboratory of Department of Education of Guangdong Province, \\ Guangzhou 510006, China \\ 3 Abastumani Observatory, Mt. Kanobili, Abastumani 0301, Georgia; O.Kurtanidze@lsw.uni-heidelberg.de (O.K.); \\ mnik@abao.kheta.ge (M.G.N.); okur@abao.kheta.ge (S.O.K.); gmelik@dtapha.kheta.georgia.su (R.C.) \\ 4 Engelhardt Astronomical Observatory, Kazan Federal University, Tatarstan 420000, Russia \\ 5 Xinjiang Astronomical Observatory, Chinese Academy of Sciences, Urumqi 830011, China; liux@xao.ac.cn \\ 6 Astrophysikalisches Institut Potsdam, An der Sternwarte 16, Potsdam 14482, Germany; gmrichter@aip.de \\ 7 Faculty of Physics and Electronic Information, LangFang Teacher's College, China; hthtwang@126.com \\ 8 Department of Astronomy, Boston University, Boston, MA 02215, USA; sasada@kusastro.kyoto-u.ac.jp \\ 9 National Astronomical Observatory, Chinese Academy of Sciences, Beijing 100864, China; aiying@nao.cas.cn \\ * Correspondence: fjh@gzhu.edu.cn
}

Academic Editors: Jose L. Gómez, Alan P. Marscher, and Svetlana G. Jorstad

Received: 14 July 2016; Accepted: 18 August 2016; Published: 30 August 2016

\begin{abstract}
In this work, we present optical R band observations of AO 0235+164 carried out during the period of November 2006 to December 2012 using the Ap6E CCD camera attached to the primary focus of the $70 \mathrm{~cm}$ meniscus telescope at Abastumani Observatory, Georgia. It shows a large variation of $\Delta R=4.88 \mathrm{mag}(14.19-19.07 \mathrm{mag})$ and a short time scale of $\Delta T_{v}=73.5 \mathrm{~min}$ during our monitoring period. When periodicity analysis methods are applied to the R-band data from both historic and our observations, periods $P_{1}=8.26 \mathrm{yr}$ and $P_{2}=0.54 \mathrm{yr}$ are found.
\end{abstract}

Keywords: galaxies; BL Lacertae objects; individual (AO 0235+164); photometry; variability

\section{Introduction}

Blazars are a special subclass of Active Galactic Nuclei (AGN) showing extreme observed properties such as high luminosity, strong and variable gamma ray emission, strong or no emission features, high and variable polarization, and superluminal motion. Blazars consist of two subclasses: BL Lacertae objects (BLs) and flat spectrum radio quasars (FSRQs). Physical classification of blazars is based on their peak frequency in their spectral energy distributions (SEDs) [1,2]. Abdo [3] extended the definition to all types of non-thermal dominated AGNs using new acronyms, such as low synchrotron peaked blazars (LSP, $\log v_{\mathrm{p}}<14 \mathrm{~Hz}$ ), intermediate synchrotron peaked blazars (ISP, $\log v_{\mathrm{p}}=14 \sim 15 \mathrm{~Hz}$ ), and high synchrotron peaked blazars (HSP, $\log v_{\mathrm{p}}>15 \mathrm{~Hz}$ ). Quite recently, we calculated the SEDs for a sample of 1425 Fermi blazars and proposed classification as follows: LSP with $\log v_{\mathrm{p}}<14 \mathrm{~Hz}$; ISP with $\log v_{\mathrm{p}}=14 \sim 15.3 \mathrm{~Hz}$; HSP with $\log v_{\mathrm{p}}>15.3 \mathrm{~Hz}$ [4].

Variability is one of the most extreme observational properties of blazars, which show variability accross almost all electromagnetic wavebands. The variations have been found to be over time scales from less than one hour to as long as years; see [5], who divided the time scales into three classes: micro-variability (intra-day variability, or IDV) with time scale $\Delta T$ being less than one day; short-term variation (STV), with $\Delta T$ being one day to several months; and long-term variation (LTV), with $\Delta T$ being longer than one year. From observations, we can see that the short-term variations are 
non-periodic, while the long-term variations in some cases are quasi-periodic, as discussed in the literature [6-17].

BL Lac AO $0235+164$ shows variability timescales from less than one hour to several years [9,18-25]. In our previous paper, variations in the UBVRI bands are $\Delta U=4.26, \Delta B=5.47$, $\Delta V=4.74, \Delta R=4.18$, and $\Delta I=3.85 \mathrm{mag}[26]$.

Raiteri et al. [27] analyzed about 25 years of observational data in optical and radio bands during the period from 1975 to 2000, and found a quasi-periodicity of the main radio (and optical) outbursts on a 5.7-year time scale. A period of $5.87 \pm 1.3$ years was found in our pervious work based on 16 years of optical observations [9], but $5.8 \pm 0.3$ years (based on $14.5 \mathrm{GHz}$ light curve), $5.7 \pm 0.3$ years (based on $8.0 \mathrm{GHz}$ light curve), and $10.0 \pm 1.3$ years (based on $4.8 \mathrm{GHz}$ light curve) are found in its radio bands [13]. This perhaps suggests the existence of a binary black hole system at its center [28,29]. It is one of the objects in our monitoring programme at Abastumani Observatory, Georgia [30-34].

In Section 2, we describe the observations; in Section 3, we show period analysis results; and in Section 4, we give discussions and conclusions.

\section{Observations}

All our observations were made using a $70 \mathrm{~cm}$ meniscus telescope at Abastumani Observatory. The observations and data reduction were described in our recent work [34]. The $R$-band light curve for AO 0235+164 is shown in red in Figure 1.

From the red points in Figure 1, we can see that AO 0235+164 is extremely variable. It shows a variation of $\Delta R \sim 4.88 \mathrm{mag}$ from $R=14.19$ to $R=19.07 \mathrm{mag}$, and a short time scale of $\Delta T_{v}=73.5 \mathrm{~min}$ during the whole observing period [34].

\section{Period Analysis}

We have compiled the optical data from the literature [9,24], and constructed an optical light curve covering a time span of 30 years, as shown in Figure 1. It is clear that the light curve is not evenly sampled. In the case that data are unevenly sampled in time series (as we have done in [33]), the Jurkevich (JV) method [6] and improved power spectral analysis (PSA) will be adopted for the possible periodicities.

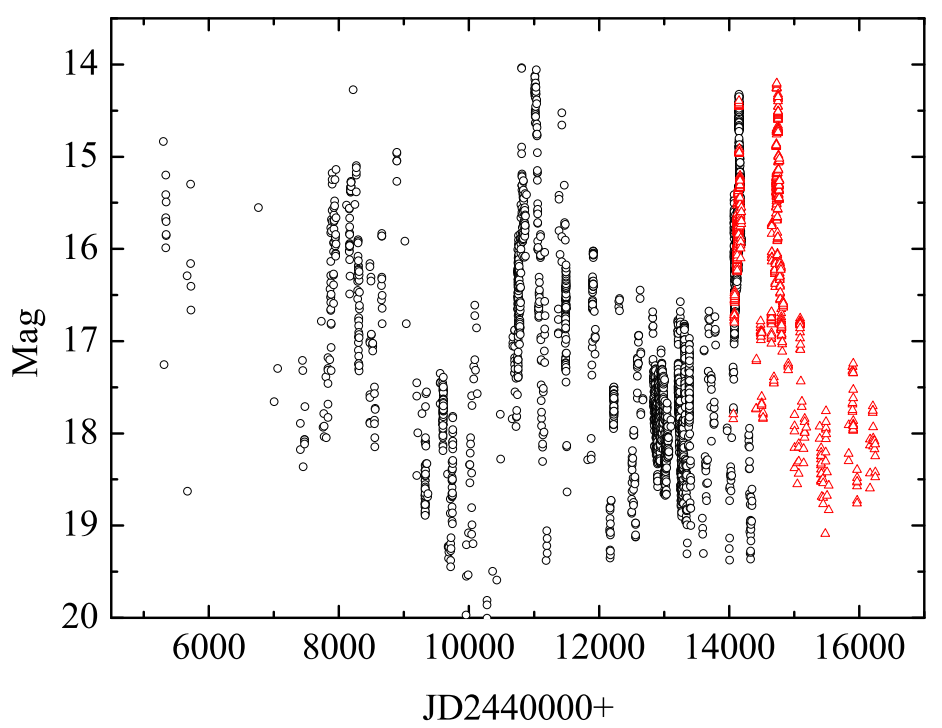

Figure 1. Historic light curve of AO 0235+164 during JD 2445300 to JD 2456247. The open circles are from the literature and the triangles are from our own observations. 
The JV method is based on the expected mean square deviation. The deviation $V_{m}^{2}(\tau)$ of a given period $\tau$ for a light curve $X\left(t_{i}\right), i=1,2, \cdots, N$ can be calculated, as described in [33,35]. If a frequency $f=1 / \tau$ is equal to the true frequency, then $V_{m}^{2}(\tau)$ reaches the minimum. The plot of $V_{m}^{2}(f)$ against $f$ is shown in the top panel of Figure 2.

Many attempts of power spectral analysis have been made to investigate the periodicity. An improved technique is the date-compensated discrete Fourier transform (DCDFT)+ the CLEANest algorithm [36,37], a least-square regression on $\sin (\omega t), \cos (\omega t)$, and constant function. The DCDFT is a powerful method for unevenly spaced data, so we adopted it to the Figure 2 light curve [37].
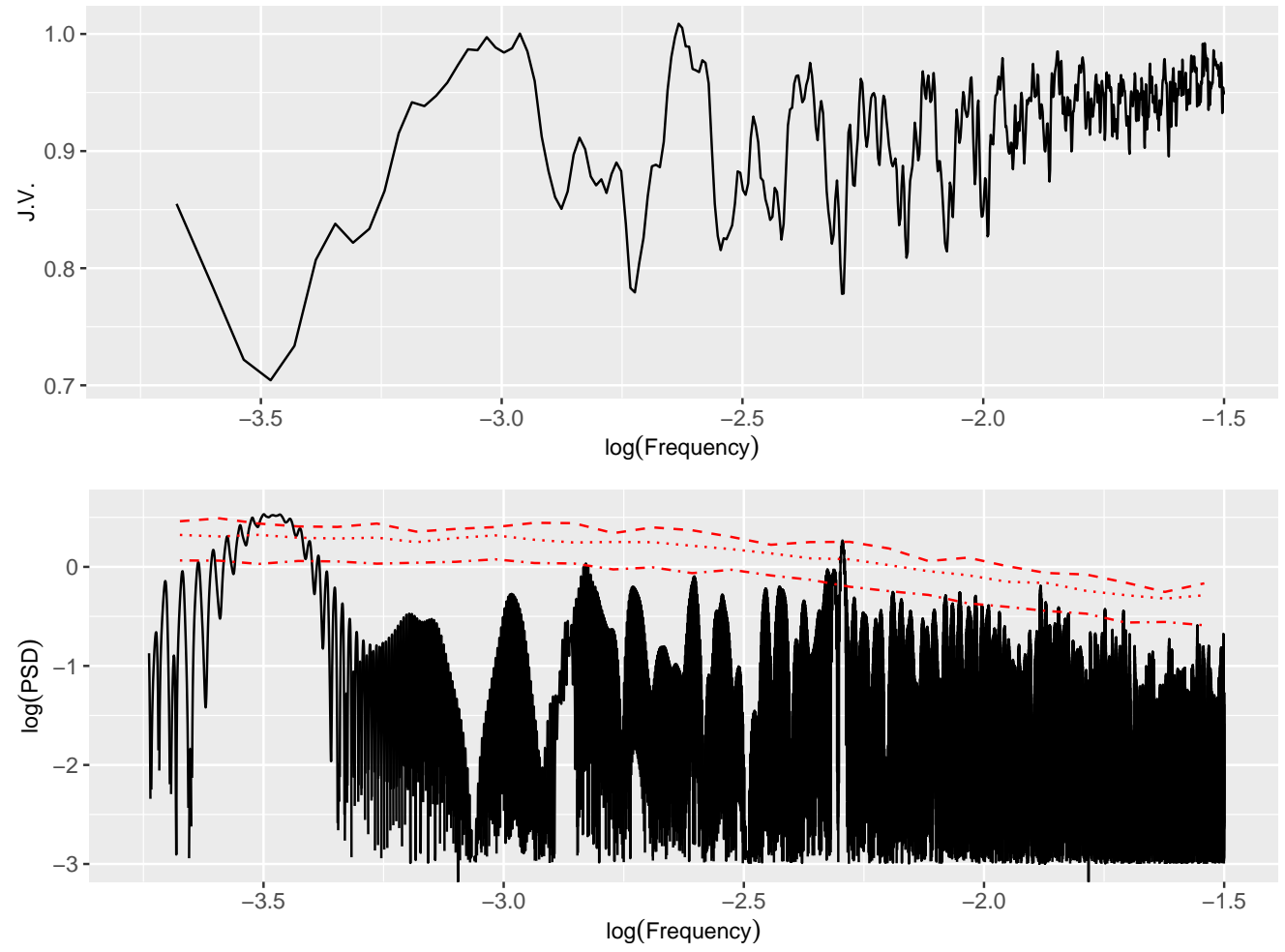

Figure 2. Top panel: Periodicity analysis results of AO 0235+164 obtained by Jurkevich (JV) method. Bottom panel: results for AO $0235+164$ by power spectral analysis (PSA) method. The curves of the false alarm probability by using the continuous autoregressive process (CAR1) method are also plotted. Two signals, $P_{1}=8.26 \mathrm{yr}$ and $P_{2}=0.54 \mathrm{yr}$, can be found with the threshold that false alarm probability $(F A P) \geq 3 \sigma$.

We assume that there are seven independent frequency components to clean the observational data; the CLEANest spectrum is shown in Figure 2. There are signs of periods: $P_{1}=8.26 \mathrm{yr}$, $P_{2}=0.54 \mathrm{yr}, P_{3}=0.85 \mathrm{yr}, P_{4}=1.99 \mathrm{yr}$, and $P_{5}=0.56 \mathrm{yr}$ in the optical light curve.

We also use the first order continuous autoregressive process (CAR1) to estimate the false alarm probability (FAP) of red noise, which CAR1 process $Y_{t}$, a model of red noise, can be given by the stochastic difference function :

$$
Y_{t}=-\frac{1}{\tau} Y_{t} \mathrm{~d} t+\mathrm{d} W_{t}
$$

Here, $\tau$ is the timescale of the CAR1 process, and $W_{t}$ is the Weiner process. The value of $\tau$ can be obtained from the light curve [38], $\tau=377_{-17}^{+54}$ days. The simulated FAP curves are also shown in Figure 2. We can find only two possible periods, $P_{1}=8.26 \mathrm{yr}$ and $P_{2}=0.54 \mathrm{yr}$, which show that threshold $F A P \geq 3 \sigma[34]$. 


\section{Discussions and Conclusions}

Variability is one of the extreme properties of BL Lacertae objects. A variation can be taken as a real one if the variability shows up in simultaneous multiwavelength observations. However, most monitoring programs are performed at a certain waveband. Romero et al. [39] introduced a variability parameter $C$ to justify a variation to be strong or not. Heidt \& Wagner [40] proposed a method to obtain a variability amplitude $A$. We also proposed that a variation can be taken as a real one if the variability is three times greater than the deviation; namely, $\Delta m_{12}=m_{1}-m_{2} \geq 3 \sqrt{\sigma_{1}^{2}+\sigma_{2}^{2}}$, where $\sigma_{1}$ and $\sigma_{2}$ are the uncertainties corresponding to $m_{1}$ and $m_{2}$. The corresponding time interval is adopted as the time scale $\Delta \mathrm{T}=\mathrm{t}_{m 2}-\mathrm{t}_{m 1}$ [41]. The time scale is also defined to be $\Delta T_{v}=\Delta F /(d F / d t)$.

AO $0235+164$ is a well-studied object. Although it was classified as a BL Lac object, the equivalent width (EW) of emission lines varies from one observational epoch to another. It is violently variable in all wavebands from radio through high-energy $\gamma$-rays [23].

At the optical bands, rapid variability was reported by many authors [20,40,42]. Schramm et al. [42] reported extreme optical variability of 1.6 mag within $48 \mathrm{~h}$. Heidt \& Wagner [40] found a variation amplitude of $6.33 \%$ per day. Romero et al. [20] reported intra-night variability with amplitudes of $A \sim 100 \%$ over $24 \mathrm{~h}$, variations of $\Delta m=0.5$ mag were detected in $\mathrm{R}$ and $\mathrm{V}$ bands within a single night, and variations up to 1.2 magnitudes occurred from night to night.

In the soft X-ray region, ROSAT detected an increase by a factor of 1.7 in about 3 days, and a decrease of a factor of 3.5 in about 13 days [43], which suggests doubling time scales of $\Delta T_{D}=1.76$ and 3.71 days, respectively.

Variability: For $\mathrm{AO} 0235+164$, its historic variation amplitude is as large as $\Delta m \sim 5.0 \mathrm{mag}[26,44,45]$. In our monitoring period, the light curve shows a variation amplitude of $\Delta R \sim 4.88$ mag, which is similar to the historically largest amplitude.

Periods: Periodicity analysis is also interesting in active galactic nuclei (AGNs). AO 0235+164 has been observed and periodicity analyzed in many works (see [34]).

Using discrete Fourier transform (DFT), Webb et al. [46] analyzed the optical light curve of AO $0235+164$ and found periods of $2.79,1.53$, and 1.29 years. Later on, we removed a linear trend from the light curve and adopted unequal-interval Fourier transform and CLEAN techniques to its normalized data, and obtained periods of 2.7 and 1.2 years. Smith \& Nair [47] found periods of 2.7 and 3.6 years in the optical band. Raiteri et al. [27] reported a 5.67 year period in the R optical light curve and 1.8, 2.8, and 3.7 years in radio bands.

In our previous papers $[9,13]$, we found periods of 2.0 years, $2.95 \pm 0.15$ years, and $5.87 \pm 1.3$ years in the optical light curve, and periods of $10.0 \pm 1.3$ years, $5.7 \pm 0.3$ years, and $5.8 \pm 0.3$ years at $4.8 \mathrm{GHz}$, 8.0 GHz, and $14.5 \mathrm{GHz}$.

Raiteri et al. [27] claimed a possible quasi-periodic occurrence of the major radio and optical outbursts of AO $0235+164$ every $5.7 \pm 0.5$ years, which resulted in the Whole Earth Blazar Telescope WEBT campaign observing the source $[48,49]$. The period analysis based on the historic and the campaign observations suggests that the period for the large outburst is $8 \sim 8.5$ years [48-51]. It is clear that the periodicity analysis results depend on the observations, which is why different works give different results.

In this work, we find that two periods $-P_{1}=8.26 \mathrm{yr}$ and $P_{2}=0.54 \mathrm{yr}-$ have $F A P \geq 3 \sigma$ in the optical light curve. Our period of $P_{1}=8.26 \mathrm{yr}$ with high confidence is consistent with the results of [48-51]. However, the periods of $P_{5}=0.54 \sim 0.56 \mathrm{yr}$ and $P_{5}=0.85 \mathrm{yr}$ have not been claimed in the literature.

The long-term period maybe caused by a binary black hole system $[7,8,14,33,34]$.

\section{Summary}

In this work, we have presented the R optical observations during the period of November 2006 to December 2012. The following results are obtained: 
(1) The largest variation $\Delta R=4.88$ mag is detected from our observations.

(2) There are two periods in the light curve, $P_{1}=8.26 \mathrm{yr}$ and $P_{2}=0.54 \mathrm{yr}$.

Acknowledgments: The work is partially supported by the National Natural Science Foundation of China (NSFC U1531245, U1431112, U11203007, 11403006, 10633010, 11173009), the Innovation Foundation of Guangzhou University (IFGZ), Guangdong Province Universities and Colleges Pearl River Scholar Funded Scheme(GDUPS)(2009), Yangcheng Scholar Funded Scheme(10A027S), and support for Astrophysics Key Subjects of Guangdong Province and Guangzhou City. The Abastumani team acknowledges financial support of the project FR/639/6-320/12 by the Shota Rustaveli National Science Foundation under contract 31/76. This work was presented in "Blazars through Sharp Multi-Wavelength Eyes", Malaga (Spain), 30 May-3 June 2016.

Author Contributions: Junhui Fan is responsible for the writing and the motivation of the paper; Omar Kurtanidze, Gotthard M Richter, Maria G Nikolashvili, Sofia O Kurtanidze, Revaz Chanishvili are responsible for the observations and data reductions; Yi Liu is for period analysis; Hongtao Wang is for the historic data collection; Mahito Sasada provides some optical data; Chao Lin is responsible for the edition of the paper and checking the references; Xiang Liu, Aiying Zhou, and Yuhai Yuan are responsible for some discussions.

Conflicts of Interest: The author declares no conflict of interest.

\section{References}

1. Padovani, P.; Giommi, P. The connection between X-ray- and radio-selected BL Lacertae objects. Astrophys. J. 1995, 444, 567-581.

2. Urry, C.M.; Padovani, P. Unified Schemes for Radio-Loud Active Galactic Nuclei. Publ. Astron. Soc. Pac. 1995, 107, 803-845.

3. Abdo, A.A.; Ackermann, M.; Agudo, I.; Axelsson, M.; Baldini, L.; Ballet, J.; Barbiellini, G.; Bastieri, D.; Baughman, B.M.; Bechtol, K.; et al. The Spectral Energy Distribution of Fermi Bright Blazars. Astrophys. J. 2010, 716, 30-70.

4. Fan, J.H.; Yang, J.H.; Liu, Y.; Luo, G.Y.; Lin, C.; Yuan, Y.H.; Xiao, H.B.; Zhou, A.Y.; Hua, T.X.; Pei, Z.Y. The Spectral Energy Distributions of Fermi Blazars. Astrophys. J. Suppl. Ser. 2016, in press.

5. Fan, J.H. Optical Variability of Blazars. Chin. J. Astron. Astrophys. 2005, 5, 213-223.

6. Jurkevich, I. A Method of Computing Periods of Cyclic Phenomena. Astrophys. Space Sci. 1971, 13, $154-167$.

7. Sillanpää, A.; Haarala, S.; Valtonen, M.J.; Sundelius, B.; Byrd, G.G. OJ 287-Binary pair of supermassive black holes. Astrophys. J. 1988, 325, 628-634.

8. Fan, J.H.; Xie, G.Z.; Pecontal, E.; Pecontal, A.; Copin, Y. Historic Light Curve and Long-Term Optical Variation of BL Lacertae 2200+420. Astrophys. J. 1998, 507, 173-178.

9. Fan, J.H.; Lin, R.G.; Xie, G.Z.; Zhang, L.; Mei, D.C.; Su, C.Y.; Peng, Z.M. Optical periodicity analysis for radio selected BL Lacertae objects (RBLs). Astron. Astrophys. 2002, 381, 1-5.

10. Ciaramella, A.; Bongardo, C.; Aller, H.D.; Aller, M.F.; De Zotti, G.; Lähteenmaki, A.; Longo, G.; Milano, L.; Tagliaferri, R.; Teräsranta, H.; et al. A multifrequency analysis of radio variability of blazars. Astron. Astrophys. 2004, 419, 485-500.

11. Wu, J.H.; Zhou, X.; Wu, X.B.; Liu, F.K.; Peng, B.; Ma, J.; Wu, Z.; Jiang, Z.J.; Chen, J.S. Optical Monitoring of BL Lacertae Object OJ 287: A 40 Day Period? Astron. J. 2006, 132, 1256-1266.

12. Ciprini, S.; Takalo, L.O.; Tosti, G. Ten-year optical monitoring of PKS 0735+178: Historical comparison, multiband behavior, and variability timescales. Astron. Astrophys. 2007, 467, 465-483.

13. Fan, J.H.; Liu, Y.; Hua, T.X.; Wang, H.G.; Wang, Y.X.; Yang, J.H.; Gupta, A.C.; Li, J.; Zhou, J.L.; Xu, S.X.; et al. Radio variability properties for radio sources. Astron. Astrophys. 2007, 462, 547-552.

14. Valtonen, M.; Kidger, M.; Lehto, H.; Poyner, G. The structure of the October/November 2005 outburst in OJ287 and the precessing binary black hole model. Astron. Astrophys. 2008, 477, 407-412.

15. Rani, B.; Gupta, A.C.; Joshi, U.C.; Ganesh, S.; Wiita, P.J. Quasi-periodic Oscillations of 15 Minutes in the Optical Light Curve of the BL Lac S5 0716+714. Astrophys. J. 2010, 719, L153-L157.

16. Qian, B.C.; Tao, J. Optical Monitoring of PKS 0735+178 from 1995 to 2001 and Its Historical Periodic Light Curve. Publ. Astron. Soc. Pac. 2004, 116, 161-169.

17. Gupta, A.C. Quasi Periodic Oscillations in Blazars. J. Astrophys. Astron. 2014, 35, 307-314.

18. Webb, J.R.; Howard, E.; Bentez, E.; Balonek, T.; McGrath, E.; Shrader, C.; Robson, I.; Jenkins, P. The 1997 Outburst of AO 0235+164: Evidence for a Microlensing Event? Astron. J. 2000, 120, 41-46. 
19. Romero, G.E.; Combi, J.A.; Benaglia, P.; Azcarate, I.N.; Cersosimo, J.C.; Wilkes, L.M. Search for intraday radio variability in EGRET blazars. Astron. Astrophys. 1997, 326, 77-86.

20. Romero, G.E.; Cellone, S.A.; Combi, J.A. Extreme intranight variability in the BL Lacertae object AO 0235+164. Astron. Astrophys. 2000, 360L, 47-50.

21. Peng, B.; de Bruyn, A.G. Evidence for Microarcsecond Structure in the BL Lacertae Object AO 0235+164. Astrophys. J. 2004, 610, 151-155.

22. Hagen-Thorn, V.A.; Larionov, V.M.; Jorstad, S.G.; Arkharov, A.A.; Hagen-Thorn, E.A.; Efimova, N.V.; Larionova, L.V.; Marscher, A.P. The Outburst of the Blazar AO 0235+164 in 2006 December: Shock-in-Jet Interpretation. Astrophys. J. 2008, 672, 40-47.

23. Ackermann, M.; Ajello, M.; Ballet, J.; Barbiellini, G.; Bastieri, D.; Bellazzini, R.; Blandford, R.D.; Bloom, E.D.; Bonamente, E.; Borgland, A.W.; et al. Multi-wavelength Observations of Blazar AO 0235+164 in the 2008-2009 Flaring State. Astrophys. J. 2012, 751, 159-178.

24. Wang, H.T. The optical periodic analysis of BL Lac object AO 0235+164. Astrophys. Space Sci. 2014, 351, 281-287.

25. Vol'vach, A.E.; Larionov, M.G.; Vol'vach, L.N.; Lähteenmäki, A.; Tornikoski, M.; Aller, M.F.; Aller, H.D.; Sasada, M. Physical characteristics of the Blazar AO 0235+164. Astron. Rep. 2015, 59, 145-155.

26. Fan, J.H.; Lin, R.G. Optical Variability and Periodicity Analysis for Blazars. I. Light Curves for Radio-selected BL Lacertae Objects. Astrophys. J. 2000, 537, 101-122.

27. Raiteri, C.M.; Villata, M.; Aller, H.D.; Aller, M.F.; Heidt, J.; Kurtanidze, O.M.; Lanteri, L.; Maesano, M.; Massaro, E.; Montagni; F.; et al. Optical and radio variability of the BL Lacertae object AO 0235+16: A possible 5-6 year periodicity. Astron. Astrophys. 2001, 377, 396-412.

28. Romero, G.E.; Fan, J.H.; Nuza, S.E. The Binary Black Hole Scenario for the BL Lacertae Object AO $0235+16$. Chin. J. Astron. Astrophys. 2003, 3, 513-525.

29. Ostorero, L.; Villata, M.; Raiteri, C.M. Helical jets in blazars. Interpretation of the multifrequency long-term variability of AO 0235+16. Astron. Astrophys. 2004, 419, 913-925.

30. Kurtanidze, O.; Nikolashvili, M.; Kimeridze, G.N.; Sigua, L.A.; Kapanadze, B.Z.; Ivanidze, R.Z. Long-term photometry of blazars at Abastumani Observatory. In Proceedings of IAU Symposium 238, Prague, Czech Republic, 21-25 August 2006; Cambridge University Press: Cambridge, UK, 2007; pp. 397-398.

31. Nikolashvili, M.; Kurtanidze, O. Long-term and intra-day variability of BL Lacertae since the last great outburst. Proc. Int. Astron. Union 2007, 238, 419-420.

32. Fan, J.H.; Kurtanidze, O.; Nikolashvili, M.G.; Gupta, A.C.; Zhang, J.S.; Yuan, Y.H. Optical Photometric Observations of $\gamma$-Ray Loud Blazars. Chin. J. Astron. Astrophys. 2004, 4, 133-142.

33. Fan, J.H.; Kurtanidze, O.; Liu, Y.; Richter, G.M.; Chanishvili, R.; Yuan, Y.H. Optical Monitoring of Two Brightest Nearby Quasars, PHL 1811 and 3C 273. Astrophys. J. Suppl. Ser. 2014, 213, 26-37.

34. Fan, J.H.; Kurtanidze, O.; Liu, Y.; Liu, X.; Yang, J.H.; Richter, G.M.; Nikolashvili, M.G.; Kurtanidze, S. O.; Wang, H.T.; Sasada, M.; et al. The Variability and Period Analysis for the BL Lac AO 0235+164. Astrophys. J. 2016, submitted.

35. Liu, Y.; Fan, J.H.; Wang, H.G.; Deng, G.G. Methods for the Quasi-Periodic Variability Analysis in Blazars. J. Astrophys. Astron. 2011, 32, 79-86.

36. Ferraz-Mello, S. Estimation of Periods from Unequally Spaced Observations. Astron. J. 1981, 86, 619-624.

37. Foster, G. The cleanest Fourier spectrum. Astron. J. 1995, 109, 1889-1902.

38. Mudelsee, M. TAUEST: A computer program for estimating persistence in unevenly spaced weather/climate time series. Comput. Geosci. 2002, 28, 69-72.

39. Romero, G.E.; Cellone, S.A.; Combi, J.A. Optical microvariability of southern AGNs. Astron. Astrophys. Suppl. Ser. 1999, 135, 477-486.

40. Heidt, J.; Wagner, S.J. Statistics of optical intraday variability in a complete sample of radio-selected BL Lacertae objects. Astron. Astrophys. 1996, 305, 42-52.

41. Fan, J.H.; Peng, Q.S.; Tao, J.; Qian, B.C.; Shen, Z.Q. Optical Observations of 3C 273 From 2000 to 2008. Astron. J. 2009, 138, 1428-1434.

42. Schramm, K.-J.; Bogeest, U.; Kuhl, D.; von Linde, J.; Linnert, M.D.; Schramm, T. The Hamburg quasar monitoring program (HQM) at Calar Alto. III. Lightcurves of optically violent variable sources. Astron. Astrophys. Suppl. Ser. 1994, 106, 349-359. 
43. Urry, C.M.; Sambruna, R.M.; Worral, D.M.; Kollgaard, R.I.; Feigelson, E.D.; Perlman, E.S.; Stocke, J.T. Soft X-Ray Properties of a Complete Sample of Radio-selected BL Lacertae Objects. Astrophys. J. 1996, 463, 424-443.

44. Rieke, G.H.; Grasdalen, G.L.; Kinman, T.D.; Hintzen, P.; Wills, B.J.; Wills, D. Photometric and spectroscopic observations of the BL Lacertae object AO 0235+164. Nature 1976, 260, 754-759.

45. Stein, W.A.; Odell, S.L.; Strittmatter, P.A. The BL Lacertae objects. Annu. Rev. Astron. Astrophys. 1976, 14, 173-195.

46. Webb, J.R.; Smith, A.G.; Leacock, R.J.; Fitzgibbons, G.L.; Gombola, P.P.; Shepherd, D.W. Optical observations of 22 violently variable extragalactic sources-1968-1986. Astron. J. 1988, 95, 374-397.

47. Smith, A.G.; Nair, A.D. Timescales of Long-Term Optical Base-Level Fluctuations in Three Classes of AGN. Publ. Astron. Soc. Pac. 1995, 107, 863-870.

48. Raiteri, C.M.; Villata, M.; Kadler, M.; Ibrahimov, M.A.; Kurtanidze, O.M.; Larionov, V.M.; Tornikoski, M.; Boltwood, P.; Lee, C.-U.; Aller, M.F.; et al. Multifrequency variability of the blazar AO 0235+164. The WEBT campaign in 2004-2005 and long-term SED analysis. Astron. Astrophys. 2006, 459, 731-743.

49. Raiteri, C.M.; Villata, M.; Capetti, A.; Heidt, J.; Arnaboldi, M.; Magazzù, A. Spectroscopic monitoring of the BL Lac object AO 0235+164. Astron. Astrophys. 2007, 464, 871-878.

50. Raiteri, C.M.; Villata, M.; Larionov, V.M.; Aller, M.F.; Bach, U.; Gurwell, M.; Kurtanidze, O.M.; Lähteenmäki, A.; Nilsson, K.; Volvach, A.; et al. Radio-to-UV monitoring of AO 0235+164 by the WEBT and Swift during the 2006-2007 outburst. Astron. Astrophys. 2008, 480, 339-347.

51. Gupta, A.C.; Cha, S.-M.; Lee, S.; Jin, H.; Pak, S.; Cho, S.; Moon, B.; Park, Y.; Yuk, I.-S.; Nam, U.; et al. Multicolor Near-Infrared Intra-Day and Short-Term Variability of the Blazar S5 0716+714. Astron. J. 2008, 136, 2359-2366.

(C) 2016 by the authors; licensee MDPI, Basel, Switzerland. This article is an open access article distributed under the terms and conditions of the Creative Commons Attribution (CC-BY) license (http://creativecommons.org/licenses/by/4.0/). 